\title{
Progression of Disease at Recurrence
}

National Cancer Institute

\section{Source}

National Cancer Institute. Progression of Disease at Recurrence. NCI Thesaurus. Code C158754.

At the time of the progression or recurrence of a disease. 